\title{
Flows and Shocks: Some Recent Developments in Symbiotic Star and Nova Research
}

\author{
J. L. Sokoloski* \\ Columbia Astrophysics Laboratory \\ E-mail: jeno@astro.columbia.edu
}

\section{Stephen Lawrence}

Hofstra University

E-mail: Stephen.S.Lawrenceehofstra.edu

\section{Arlin P. S. Crotts}

Columbia Department of Astronomy

\section{Koji Mukai}

Goddard Space Flight Center and University of Maryland Baltimore County

E-mail: Koji.Mukai@nasa.gov

How — and how efficiently — do white dwarfs (WDs) accrete and expel material? The answers to these questions have bearing on binary stellar evolution and the production of type Ia supernovae, the physics of accretion disks and jets, and our understanding of stellar eruptions as we enter the golden age of time-domain astrophysics. Optical observations have contributed crucial information about accreting WDs and nova eruptions for more than half a century. In the past decade, however, observations at the more extreme ends of the electromagnetic spectrum have driven a series of breakthroughs. X-ray and UV observations of WDs that accrete from red giants hint at the existence of a heretofore hidden population of these objects. Whereas a WD that accretes from a red giant and maintains quasi-steady shell burning on its surface is very likely to show the distinctive 'symbiotic phenomenon' in its optical spectrum, a similar WD without shell burning might only reveal the interaction with its companion in the UV and/or X-rays. Furthermore, these non-burning symbiotic stars may be as numerous as burning symbiotics, and afford a particularly good view of WDs that drive powerful jets. Regarding nova eruptions, the Fermi satellite showed that they are transient $\mathrm{GeV} \gamma$-ray sources and therefore capable of particle acceleration. For novae in cataclysmic variables, this implicates internal shocks. Other signatures of shocks include thermal X-rays and non-thermal radio emission, and a substantial fraction of optical emission may be shock-powered in the early phase of novae. Radio (V959 Mon) and HST (V959 Mon and T Pyx) images of nova shells within a few years of their respective eruptions suggest that internal shocks commonly arise as a fast flow or wind collides with a slower flow that is concentrated in an equatorial ring. The flow structure within the ejecta and the properties of its internal shocks are also providing new constraints on the ejection mechanism for nova remnants, the origin of features in optical light curves, dust formation, and particle acceleration in dense environments. In both symbiotic stars and nova eruptions, emission from shocks enhances the degree to which multiwavelength observations probe the inflows and outflows from accreting WDs.

Accretion Processes in Cosmic Sources - APCS2016 -

5-10 September 2016,

Saint Petersburg, Russia

${ }^{*}$ Speaker. 


\section{Introduction: shocks point the way in symbiotic stars and novae}

During the past 5 to 10 years, multiwavelength observations and theoretical studies of symbiotic binary stars and nova eruptions have led to some surprising developments in these fields. In both symbiotic binaries and novae, the key findings are related, at least in part, to flows, shocks, and the high-energy emission that results.

Symbiotic stars are wide, interacting binaries in which a compact object accretes from a redgiant $(\mathrm{RG})$ companion. Orbital periods range from years to decades, and binary separations range from AU to tens or even hundreds of AU. Here, we restrict our discussion to symbiotics that contain white dwarf (WD) accretors. In some such symbiotic stars, the interaction is powered solely by the release of gravitational potential energy as matter falls onto the WDs. In others, the temperature and luminosity of the WD indicate that the interaction is powered primarily by the quasi-steady burning of hydrogen-rich fuel in a shell on the WD surface (see [58] for a compilation of WD temperatures and luminosities). The degree to which this shell burning is residual burning from past novae, the result of accretion at a high rate, or a combination of both is not yet evident ${ }^{1}$. But recent observations at radio through X-ray wavelengths reveal that the presence or absence of shell burning on the WD determines the characteristics of a symbiotic at every waveband. Xrays are particularly valuable for diagnosing the inner accretion flow. Lastly, symbiotics without shell burning, which have been difficult to identify in the optical, are worth searching for in the $\mathrm{X}$-ray and ultraviolet (UV) wavebands. This heretofore hidden population offers the opportunity to investigate wind-fed accretion disks with radii of $\sim 10^{12}$ to $\sim 10^{13} \mathrm{~cm}$, their jets, and the evolution and statistics of wide, interacting binaries - including the production of type Ia supernovae.

Nova eruptions occur on accreting WDs in wide, symbiotic binaries and in the much tighter cataclysmic variables (CVs). Since 2010, the Fermi Gamma-Ray Space Telescope has astonished the astronomical community with its finding that novae constitute a new class of $\mathrm{GeV} \gamma$-ray sources (e.g., $[1,2])$. It turns out that nova events generate $\gamma$-rays both when the eruptive WD is embedded within the wind of a red-giant companion, and when it is not. As we discuss below, the hunt for the origin of these $\gamma$-rays has exposed the degree to which the outflows from novae consist of multiple, distinct flows, and the crucial role of powerful shocks in almost all aspects of novae.

\section{Symbiotic stars with and without shell burning}

Energetics. The luminosity of an accreting WD with a red-giant companion is set chiefly by whether or not accreted material burns quasi-steadily in a shell on the surface of the WD. If material in the WD envelope burns at a rate slightly below that given by the core mass-luminosity relation $[69,88]$, then the luminosity of the WD is $L_{\mathrm{WD}}$ (with burning) $\sim 10^{3} L_{\odot}$ for low- to moderate-mass WDs [113]. If, on the other hand, shell burning is absent, the luminosity of the WD is roughly that generated by accretion,

$$
L_{\mathrm{WD}}(\text { without burning }) \approx G M_{\mathrm{WD}} \dot{M}_{\mathrm{WD}} / R_{\mathrm{WD}}
$$

\footnotetext{
${ }^{1}$ Decades of post-nova shell burning in the symbiotic slow novae suggest that residual burning can persist for longer in these wide binaries than in cataclysmic variables, perhaps because avoidance of common envelope interactions during the nova eruption allows more of the envelope mass to remain on the WD.
} 


$$
\approx 10 L_{\odot}\left(\frac{M_{\mathrm{WD}}}{0.6 M_{\odot}}\right)\left(\frac{\dot{M}_{\mathrm{WD}}}{10^{-8} M_{\odot} \mathrm{yr}^{-1}}\right)\left(\frac{R_{\mathrm{WD}}}{10^{9} \mathrm{~cm}}\right)^{-1}
$$

where $M_{\mathrm{WD}}$ is the mass of the $\mathrm{WD}, \dot{M}_{\mathrm{WD}}$ is the rate of accretion onto the $\mathrm{WD}$, and $R_{\mathrm{WD}}$ is the radius of the WD. As we will see below, it is not just the WD luminosities that distinguish WD-plus-RG interacting binaries with and without shell burning; the two different sources of power for the WD create observational differences across the electromagnetic spectrum.

Optical spectra. Because symbiotic stars have traditionally been defined and identified by their optical spectra, it is worth starting our discussion with the impact of shell burning (or the lack thereof) on the optical emission. In a CV, the accretion disk usually dominates the optical emission whether or not shell burning is present, because most light from the WD photosphere is radiated shortward of the optical. In a WD-plus-RG interacting binary, on the other hand, the donor is quite bright, and the wind from the RG reprocesses some of the UV/soft-X-ray emission from the burning WD into the optical. Thus, if the WD is hot and luminous due to shell burning, the optical emission is frequently dominated by light from the red giant donor and/or the ionized wind nebula [92]. In particular, the strength of the optical line and recombination-continuum emission from the nebula depends on the luminosity (and to some extent temperature) of the accreting WD, which in turn depends mainly upon whether hydrogen-rich fuel is burning quasi-steadily on its surface. With ongoing burning, the temperature of the WD sits above $\sim 10^{5} \mathrm{~K}$, and the luminous WD ionizes a large portion of the nebula. The nebula then generates the strong, high-ionization state optical emission lines that are the hallmark of classical symbiotic stars (e.g., [33, 55, 48] and references therein). Without shell burning on the WD, a predominantly neutral - and therefore faint nebula allows photospheric emission from the red giant, and in some cases the accretion disk, to dominate the optical spectrum. In this case, the optical spectrum has only weak, if any, emission lines. Extreme examples of such systems include the symbiotic recurrent novae (such as $\mathrm{T} \mathrm{CrB}$ in its normal, quiet state); the well-known jet-producing binaries CH Cyg, R Aqr, and MWC 560; and systems with very hard X-ray emission such as RT Cru, V648 Car, and SU Lyn (Fig. 1 shows the UV/optical spectrum of SU Lyn, from [53]). WD-plus-RG interacting binaries in this state have sometimes been referred to as weakly symbiotic or symbiotic-like binaries. WD-plus-RG binaries without shell burning, however, are not necessarily weakly interacting.

What's in a name? Although WD-plus-RG interacting binaries without quasi-steady shell burning on the WD often lack the strong optical emission lines that motivated the original definition of symbiotic stars, we argue that it is nevertheless appropriate and useful to include such systems among the so-called symbiotic stars ${ }^{2}$. As in past work (e.g., [32, 43, 53]), we therefore define a WD symbiotic system as a binary in which a red giant transfers enough material to a WD for the interaction to produce an observable signal at some waveband. Considering WD-plus-RG binaries with and without shell burning together is meaningful in part because the range of accretion rates in systems without shell burning $\left(\sim 10^{-10}-\sim 10^{-7} M_{\odot} \mathrm{yr}^{-1}\right.$ (e.g., $\left.[24,97,16,43]\right)$ overlaps the range of rates expected for systems with shell burning $\left(\sim 10^{-8}-\sim 10^{-7} M_{\odot} \mathrm{yr}^{-1}\right.$, depending on

\footnotetext{
${ }^{2}$ Though counter-intuitive to extend the definition of symbiotic stars to objects that do not show much of the optically defined "symbiotic phenomenon" [47], we hope readers will agree that it is more natural than introducing a new name for a what is almost certainly a transient state. Additionally, the name WD-plus-RG interacting binaries is cumbersome and obscures the connection to past research on symbiotic stars.
} 


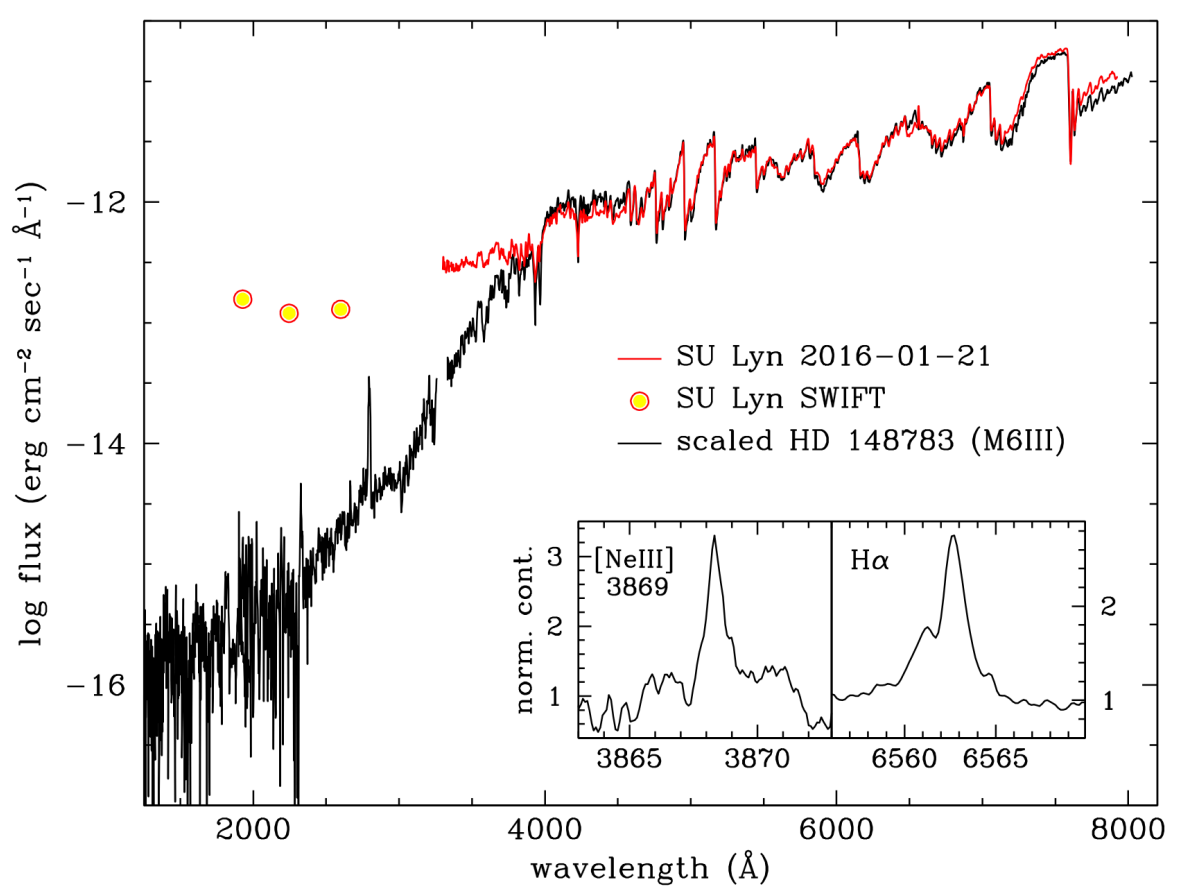

Figure 1: UV through optical spectrum of non-burning symbiotic star SU Lyn, reproduced with permission from [53]. The yellow UV points are from Swift/UVOT, and they show that the accreting WD becomes apparent in the UV. The black curve shows IUE and optical spectra of a standard MIII star, for comparison. Longward of about $4000 \AA$, the low-resolution optical spectrum of SU Lyn is difficult to distinguish from that of a normal red giant. The insets show faint emission lines (of H $\alpha$ and [Ne III] $3869 \AA$ ) detected with a high-resolution optical spectrum. SU Lyn demonstrates how difficult it is to identify such systems with low-resolution optical spectroscopy alone, even though the WD in SU Lyn accretes at a rate of more than $10^{-9} M_{\odot} \mathrm{yr}^{-1}[53]$.

WD mass [113], or even lower if the shell burning is residual burning from a recent nova). For WD-plus-RG binaries without shell burning, however, the interaction is normally more apparent in X-ray spectra and optical-UV fast photometry than in optical spectra (see below). In fact, at X-ray wavelengths and in optical-UV fast photometry, signatures of binary interaction can be stronger in non-burning than in burning symbiotics. Finally, because shell burning on the WD is almost certainly a transient phenomenon, each WD-plus-RG interacting binary is likely to spend some time with and some time without shell burning. Shell burning may even turn on and off more than once. We thus refer to WD-plus-RG interacting binaries with and without shell burning on the surface of the WD as burning and non-burning symbiotics, respectively.

$\mathrm{X}$-rays: revealing the inner accretion flow. Looking beyond the optical spectrum, the presence or absence of quasi-steady shell burning on the WD also dictates to a large extent the nature of X-rays from a symbiotic star. For example, some non-burning symbiotics emit highly absorbed $\mathrm{X}$-rays with energies greater than several $\mathrm{keV}$ and as high as tens of $\mathrm{keV}$ (which we refer to as hard X-rays). When such binaries are nearby, their hard X-ray emission can be bright enough to reveal flickering-type variations on time scales of minutes to hours, and spectra that are well 
modeled as isobaric cooling flows (as in [52]). This X-ray spectral component was referred to by [43] as $\delta$-type $\mathrm{X}$-ray emission, and it most likely emanates from an accretion-disk boundary layer (e.g., [41, 32, 16, 43, 53, 28]). Evidence for a boundary-layer origin includes: 1) rapid, stochastic brightness variations like those for the boundary layers of CVs; 2) cooling flow spectra, like those expected from flows onto the surface of a WD (and observed in CVs (e.g., [71]); 3) high intrinsic, partial-covering absorption, indicating that the source of hard X-rays is small and located deep within the nebula and/or disk wind; and 4) a lack of detectable modulation of the X-ray brightness at the WD spin period, indicating that the X-rays are unlikely to emanate from magnetic accretion columns (see [54] for a review of X-ray properties of accreting WDs). Although [14] have suggested magnetic accretion as the origin of hard X-rays in at least one non-burning symbiotic (RT Cru), we contend that the lack of spin modulations in the hard X-ray emission from all nonburning symbiotics supports the boundary-layer interpretation for the class as a whole. Burning symbiotics, in contrast, do not seem to radiate hard X-ray emission (with energies greater than a few $\mathrm{keV}$ ), perhaps because the high flux of FUV photons from the luminous, hot WDs Comptoncools the boundary layers out of the X-ray regime (as described in general terms in [17]). So, whereas searches for red giants with strong optical emission lines preferentially uncover burning symbiotics, searches for red giants with hard X-ray emission would preferentially (or exclusively) uncover symbiotics without shell burning.

Softer X-rays from shell burning, jets, and colliding winds. If a WD with shell burning is massive enough for photospheric temperatures to reach at least several hundred thousand degrees, burning symbiotics emit supersoft X-ray emission (all photons below $1 \mathrm{keV}$ and most below $0.4 \mathrm{keV}$ ), which [59] dubbed $\alpha$-type X-ray emission. Examples of symbiotics with such emission include AG Dra, RR Tel, and StH $\alpha 32$ [59, 67]. Such supersoft X-rays are only detectable if the column of absorbing material is fairly low. Both burning and non-burning symbiotics are able to produce so-called $\beta$-type thermal X-ray emission, in which most photons have energies less than approximately 2 or $3 \mathrm{keV}$. The lower column of absorbing material compared to $\delta$-type $\mathrm{X}$-ray emission, the lack of minute-to-hour time scale variability, and the lower plasma temperatures than typically found in boundary layers all suggest that $\beta$-type emission arises from colliding winds [59, 43, 63] or within collimated jets (e.g., [30, 18, 31, 62, 29, 102]). R Aqr and CH Cyg provide that most dramatic examples of X-ray emission from shock-heated plasma within WD jets (Fig. 2). For symbiotics that are faint in the X-rays, it is sometimes difficult to distinguish between $\beta$-type and $\delta$-type X-ray emission [43]. But even if it is not always clear whether moderate-energy $\mathrm{X}$-rays come from colliding winds, a jet, an accretion-disk boundary layer, or magnetic accretion columns, observations with Swift, INTEGRAL, Chandra, and most recently, NuSTAR have made it clear that the X-ray emission from symbiotic stars is complex, variable, both soft and hard, and dependent upon the presence or absence of shell burning.

Disk flickering in the UV. Accretion in CVs, X-ray binaries, and active galactic nuclei leads to stochastic brightness variations on time scales from roughly the dynamical time near the inner edge of the disk to viscous time scales throughout the disk [96]. UV light curves from Swift/UVOT have now shown that - as long as no shell burning is present to hide the disk - symbiotic stars fit this same pattern. The classic accretion signature of large-amplitude flickering (fractional rms amplitude greater than about 10\%) was not initially found to be pervasive in symbiotic stars because early searches were performed in the optical (e.g., [93, 23]). Although many symbiotics probably 

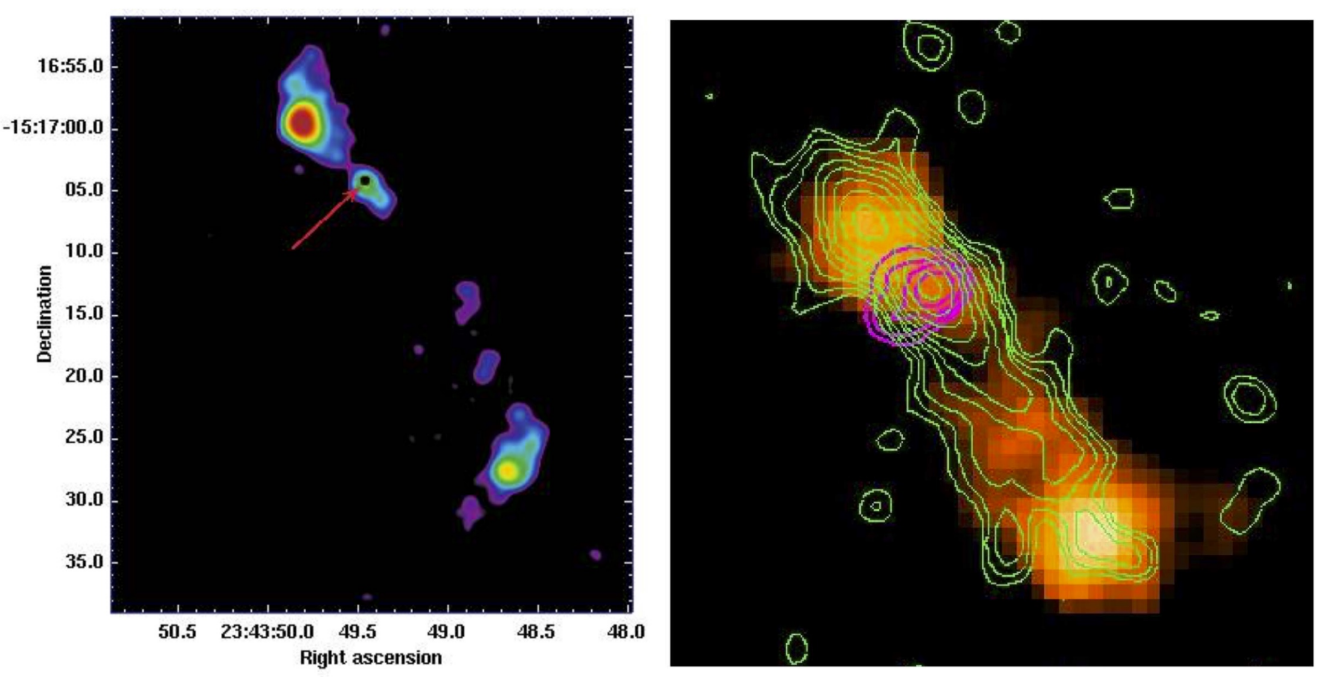

Figure 2: Chandra images of the X-ray jets from non-burning symbiotics R Aqr (left) and CH Cyg (right), reproduced from [31] and [29]. In the R Aqr image, the 0.2 to $3.5 \mathrm{keV} \mathrm{X-rays} \mathrm{were} \mathrm{smoothed,} \mathrm{and} \mathrm{the} \mathrm{red}$ arrow indicates the location of the central binary. In the $\mathrm{CH}$ Cyg image, green contours show the radio flux density at $5 \mathrm{GHz}$, magenta contours show the hard, 6-7 keV X-rays, and the yellow and orange image shows the location and strength of soft $\mathrm{X}$-rays.

contain accretion disks whose inner regions are similar to disks in CVs [38, 50, 12], the disk is not usually a major contributor to the optical light. Moving to the UV, where the red giant makes a much smaller contribution, enabled [43] to detect disk flickering from a larger fraction of symbiotics than [93] did in the optical. Moreover, based on a comparison between UV variability and X-ray spectral properties, they concluded that symbiotics without shell burning had a much greater propensity to generate large-amplitude UV flickering. In symbiotics with shell burning, UV disk light is outshined by emission from the ionized nebula - which does not generally vary on time scales of minutes to hours. Supporting the idea that flickering from the accretion flow is easier to detect if the WD does not support shell burning, all of the symbiotics known to have strong optical flickering are non-burning symbiotics (e.g., CH Cyg, V407 Cyg, RT Cru, MWC 560, and symbiotic recurrent novae such as RS Oph and T CrB; [13, 94, 95, 116, 115]).

Finding the accretion structures in symbiotic stars. Therefore, by moving beyond the optical band, [43] (and others) identified robust techniques for detecting the accretion disks in at least some symbiotics. One can probe the accretion flow by observing in the hard X-rays and/or in the UV time domain. Additionally, Fig. 1 and work by [79] show that even without variability information, one can identify red giants with UV excess as candidate non-burning symbiotics. Conversely, these same authors found that seeing a classic signatures of accretion - in this case UV or optical disk flickering, or hard X-ray emission - furnishes strong evidence that a given symbiotic is powered by accretion alone rather than shell burning. Another consequence of these findings is that there is no evidence that the inner accretion flows in symbiotics and CVs are wildly different; accretion is just usually hidden in symbiotics with shell burning.

Radio emission from burning vs non-burning symbiotics. Completing our examination 
of the observational signatures of burning and non-burning symbiotics across the electromagnetic spectrum, clear disparities exist between the radio brightnesses of the two types of symbiotics. In a seminal series of papers, $[82,83,84]$ found that most of the symbiotic stars they detected with the Very Large Array (VLA) had radio flux densities on the order of mJy, consistent with free-free emission from the ionized wind of the red giant. Because their target lists consisted primarily of objects with strong optical emission lines, the majority of the sources they detected were likely burning symbiotics. When [111] specifically targeted 11 non-burning symbiotics for radio observations, on the other hand, she found that about half of these objects had radio flux densities of approximately $10 \mu \mathrm{Jy}$ or less (T CrB, ER Del, CD -27 8661, TX CVn, MWC 560, CD 283719 , and BD -21 3873). The others on her target list (NQ Gem, UV Aur, ZZ CMi, and Wray 151470) had flux densities of a few tenths of a mJy (but with no indication that these sources are particularly distant). The finding that non-burning symbiotics tend to have very faint radio emission is consistent with the optical emission lines from these sources being weak, and the ionized regions of the red-giant winds being small compared to those in burning symbiotics. In terms of diagnosing the source of the WD's power, a luminosity of $L_{\mathrm{WD}} \sim 10^{3} L_{\odot}$ or greater is a compelling sign that shell burning is the source of power; it can, however, be challenging to measure lwd (especially if UV observations are not available). That non-burning symbiotics generally have much weaker radio emission than burning symbiotics furnishes a more easily attainable observational diagnostic of the burning status.

Fortuitously, the low quiescent-state radio luminosity of non-burning symbiotics might also allow radio emission to be used as an effective probe of transient, bipolar outflows. For instance, [40] discovered a major radio brightening during the 2016 optical high state of MWC 560 [56]. They concluded that the radio brightened as a result of an increase in the power of the well-known (possibly bipolar) outflow in concert with the rise in the accretion rate onto the WD.

Selection bias and the missing population of interacting binaries. Considering our juxtaposition of burning and non-burning symbiotics, it becomes evident that optical spectroscopic surveys, which excel at finding burning symbiotics, almost certainly miss many (or even most) non-burning symbiotics. Recent and on-going optical spectroscopic surveys are adding to the number of symbiotics and candidate symbiotics, both in our galaxy (e.g., $[9,10,106])$ and in nearby galaxies [49]. But with a bias toward finding burning symbiotics, these data could tempt us into drawing mistaken conclusions about number densities and birth rates of symbiotic stars, as well as the distributions of their properties. For example, if shell burning is easier to establish and maintain on low-mass WDs (e.g., [113]), symbiotics identified by strong lines in their optical spectra would tend to contain low-mass WDs, or higher rates of mass transfer. Indeed, compilations of WD mass estimates for optically selected symbiotics do suggest that this sample on average has low-mass WDs (e.g., [48]). Conversely, symbiotics with hard X-ray emission are more likely to contain high-mass WDs (e.g., $[41,42,16])$ and/or lower rates of mass transfer. In the most extreme case, a reliance on optical spectroscopy could mean we have missed almost an entire population of interacting binary stars - the non-burning symbiotics. Work by [57] suggesting that an appreciable fraction of the so-called Galactic ridge X-ray emission could be due to WDs accreting from red-giant companions; past detections of X-rays from red giants by [105]); and estimates of the space density of non-burning symbiotics by [53] all raise the intriguing possibility that the population of non-burning symbiotics is significant. Finally, because nearby non-burning symbiotic stars 
power some of the most dramatic WD jets (e.g., R Aqr, CH Cyg; Fig. 2), unveiling the population of non-burning symbiotics could be particularly exciting for the study of astrophysical disks and jets. We are currently laying the groundwork for moving beyond optical spectroscopy to search for this missing population.

\section{Novae: colliding flows explain $\gamma$-rays and more}

Two distinct flows. Turning to novae, we now direct our attention from WD inflows to outflows. At a fundamental level, the ejecta from novae appear to consist of two main components: a slow, dense outflow with a maximum velocity of less than about $1000 \mathrm{~km} \mathrm{~s}^{-1}$ and a fast outflow or wind with a maximum velocity of several thousand $\mathrm{km} \mathrm{s}^{-1}$. As a nova remnant expands, the slow flow is observed as a dense core in radio or optical images (e.g., HST images of V959 Mon and T Pyx, below), whereas the fast flow often takes the form of more extended, bipolar lobes. In addition to the two novae discussed below, the $\gamma$-ray bright nova V339 Del affords a nice example of this behavior $[81,19]$. Because the rapidly expanding outer structures tend to fade within a few years, many of the optical images of old nova shells (observed more than a decade after the novae eruption (e.g., [20, 22] may record principally the slow-moving component of the ejecta. Supporting this idea, these old shells regularly show flow speeds of less than $1000 \mathrm{~km} \mathrm{~s}^{-1}$. In terms of optical line profiles, [19] note that $[35,74,75,81]$ all conclude that high-velocity wings on emission lines detected in early spectra of CO novae "originate in low-density, outlying gas, and that the lower velocity given by the line core is representative of the expansion rate of the bulk of the ejecta." Critically, the ejecta do not consist of a structure that expands uniformly from $t_{0}$ (the time of the thermonuclear runaway; TNR) - rather, the fast flow plows through and/or around the slow flow, getting shaped by it and giving rise to strong shocks.

Equatorial rings. Within the slower component of the ejecta, the density is often enhanced toward the equatorial plane (e.g., $[72,7,15,98,8]$ ). This equatorial torus presumably shapes the faster component of the ejecta into a bipolar morphology. Evidence for slow, equatorial rings and faster, bipolar outflows comes from the complex profiles of optical emission lines (e.g., [27, 101, $21,77,90,78])$. Optical and radio imaging support the idea that equatorial rings and bipolar lobes are common (e.g., [20,114] and the HST images below). Minor features such as polar blobs, polar rings, general clumpiness, and an early 'puff' of ejecta are also often observed, but the most energetically important components of the ejecta appear to be the slow and fast flows, which tend to produce equatorial rings and bipolar lobes, respectively. Furthermore, [8] used radio observations of the $\gamma$-ray bright nova V959 Mon to infer that collisions between a dense equatorial torus and a faster flow led to shocks that accelerated particles, explaining the stunning recent discovery that many normal novae produce GeV $\gamma$-rays $[2,5]$.

Generation of $\gamma$-rays. Moreover, the scenario for $\gamma$-ray production inferred for V959 Mon by [8] could be broadly applicable. Relativistic particles that generate $\gamma$-rays from classical novae (either via inverse-Compton scattering or the decay of neutral pions) are almost certainly accelerated in shocks. Although some nova-producing WDs that are embedded in the wind of a red-giant companion (which we refer to as embedded novae) produce $\gamma$-ray emission via external shocks between the ejecta and the pre-existing circumbinary material (e.g., in V407 Cyg [1, 60, 65] and V745 Sco [4, 68, 66]), most $\gamma$-ray bright novae are not embedded in such dense environments [2]. 
Thus, the location and properties of shocks within nova shells are crucial for understanding how classical (non-embedded) novae generate $\gamma$-rays. With its fortuitously high inclination [70, 91, 78], V959 Mon displayed radio synchrotron-producing shocks between a bipolar flow extending to the east and west, and a less extended equatorial torus aligned north-south and viewed from the edge [8]. But we know that equatorial rings and shocks are both common in classical novae. As we discussed above, imaging at various wavelengths and optical emission-line profiles frequently reveal equatorial rings. X-ray emission with photon energies greater than approximately $1 \mathrm{keV}$, and radio synchrotron emission (e.g., [36, 109, 110]), reveal shock-heated gas (e.g., [51]) and particles accelerated in shocks, respectively. Thus, the ingredients that led to gamma-rays in V959 Mon are common. Most novae could therefore perhaps generate $\gamma$-rays as in V959 Mon.

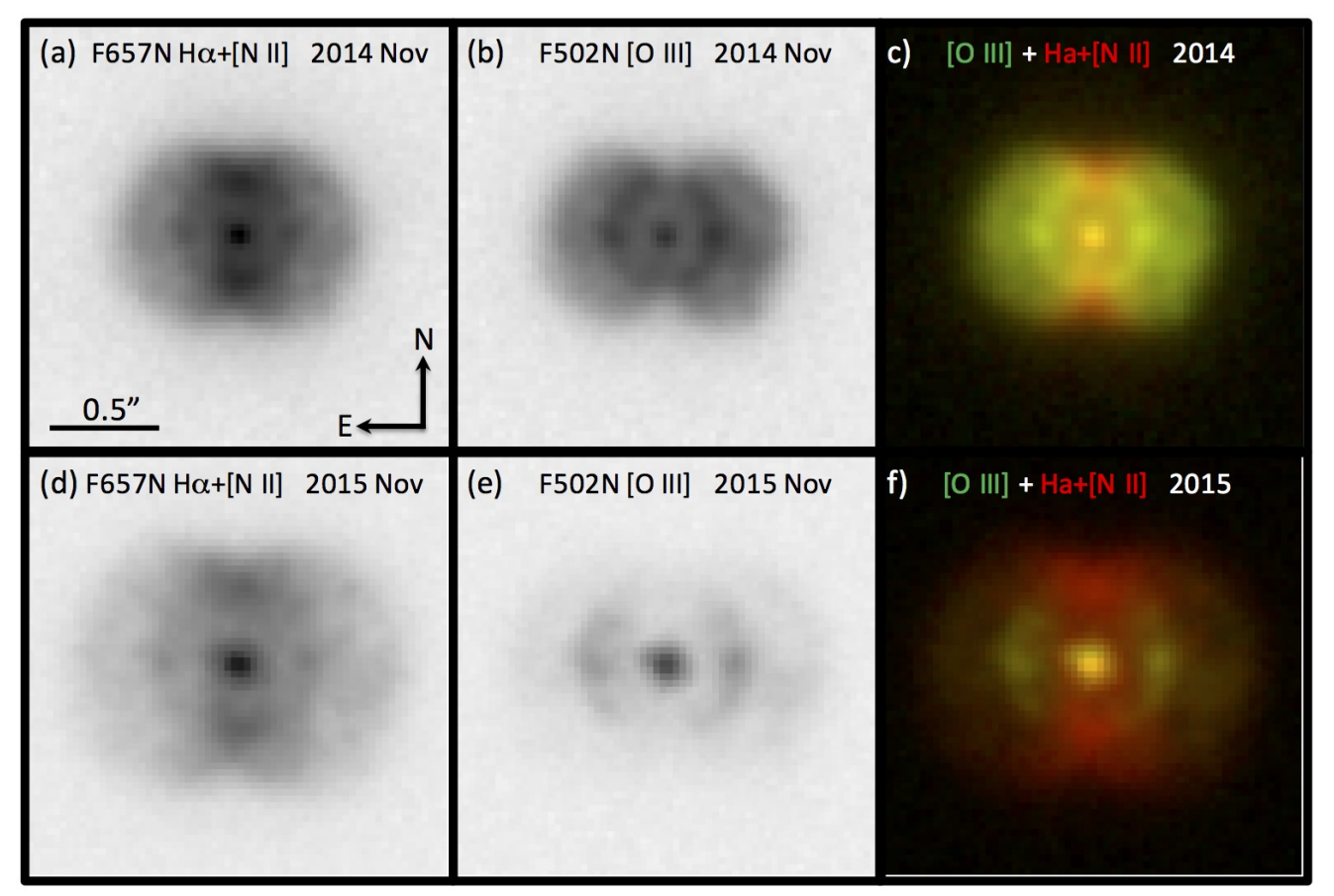

Figure 3: HST/WFC3 images of V959 Mon, one of the first classical nova to be detected by Fermi. Images have been drizzled to $0.03^{\prime \prime}$ pixel scale. All frames are 64 pixels $=1.92^{\prime \prime}$ square, $\mathrm{N}$ up, with identical logarithmic intensity scaling and stretch (pegged to brightest pixel in F657N in 2014). (a) F657N 2014 November. (b) F502N 2014 November. (c) RGB color frame mapping G channel to F502N 2014, R channel to F657N 2014 and B channel left blank. (d) F657N 2015 November. (e) F502N 2015 November. (f) As with panel (c), but for the 2015 epoch.

HST observations of V959 Mon. To examine the dominant structures in the ejecta from V959 Mon more clearly, we turn to HST images and imaging spectroscopy. On 2014 November 21 and then 2015 November 30, HST observed V959 Mon with the WFC3 camera (and on 19 December 2014 and 21 December 2015 with STIS; program 13715 [PI: Sokoloski]). With an 
initial discovery date for the nova (in the $\gamma$-rays) of 2012 June 19 ([2]; see also [3]), the HST observations captured the state of the remnant approximately 2.5 and 3.5 years after the start of the outburst. Fig. 3 shows HST/WFC3 images of V959 Mon through the F657N filter ( $\mathrm{H} \alpha+[\mathrm{NII}]$; left column) and F502N filter ([OIII]; right column).

The $[\mathrm{H} \alpha+N I I / \mathrm{F} 657 \mathrm{~N}]$ images of V959 Mon are dominated by $\mathrm{H} \alpha$ emission, which traces dense, ionized gas. They show the central binary (the unresolved central point source), an overall bipolar structure with a major axis in the east-west direction, and four knots lying on a circular feature that spans the minor axis (north-to-south) of the remnant. The outermost, bipolar shape is roughly consistent with the morphology that [78] inferred from the profiles of optical emission lines and that [8] detected in their VLA images on day 126. The STIS spectroscopic image indicates that the eastern lobe is somewhat blueshifted (tilted slightly toward the observer) and the western lobe is somewhat redshifted (tilted slightly away from the observer). The two strongest knots, along the north-south axis, are consistent with limb-brightened emission from the edge-on equatorial torus that dominated the radio images on day 615 [8]. Additionally, the kinematics of the central circular structure from our two epochs of $H S T /$ STIS imaging spectroscopy suggest that the circular ring in the WFC3 images is actually a ring plus caps or a 3-dimensional spherical shell of material expanding with a velocity of approximately $1000 \mathrm{~km} \mathrm{~s}^{-1}$ [99]. Thus, the HST observations show that the equatorial torus that presumably shaped the faster, bipolar flow is part of a more complete, spherical core. Interestingly, by three and a half years into the eruption, $\mathrm{H} \alpha$ (and [OIII]) emission from the outer, fast flow had almost completely faded, leaving a remnant that was much more spherically symmetric than the original, bipolar morphology. We identify this spherical core with its equatorial density enhancement - as the slow component of the ejecta.

Compared to the $\mathrm{H} \alpha$ emission, [OIII] emission from V959 Mon traces more diffuse gas. Detectable levels of [OIII] emission emanated from the same features as $\mathrm{H} \alpha$ - except for the dense, edge-on equatorial torus (see Fig. 3; right side). The extent of the [OIII] remnant in 2014 November (day 875 after $\gamma$-ray detection) along the major axis was $1.05^{\prime \prime}$ (measured from locations that were $10 \%$ of the peak flux). The spherical shell and two knots to the east and west of the central point source that were quite faint in $\mathrm{H} \alpha$ outshined most other features in [OIII]. And some hints of tails extending outward from the two [OIII] knots are also present. In 2014 December, STIS also detected $\mathrm{Ne}[\mathrm{V}](\lambda 3426 \AA)$ emission from the regions of the [OIII] knots, whose emission was Doppler shifted in the same sense as the larger lobes. As the [OIII] remnant expanded between 2014 and 2015, the inner spherical shell took on the appearance of two arcs. Even more so than in the $\mathrm{H} \alpha$ images, the outer lobes almost completely faded in [OIII] by the 2015 November. We describe the HST WFC3 and STIS observations of V959 Mon in detail in a forthcoming paper [99].

HST observations of T Pyx. Moving on to another nova-producing binary, HST observations of the recurrent nova T Pyx highlight the similarities among even quite disparate novae. We observed T Pyx with HST/WFC3 on 2012 July 12; 2013 January 30, June 19, July 15, and September 17-18; and 2014 July 10. We also obtained 9 observations with HST/STIS (gratings G430L and G750L) spanning this same time period (through programs 12448, 13400, and 13796 [PI: Crotts]). Although T Pyx is generally considered to be a quite unique nova-producing binary, with its short orbital period (of $1.8 \mathrm{hr}$; e.g. [73]) and high rate of mass transfer [34, 104], its ejecta contain the same basic components as that of V959 Mon. Light-echoes and flash ionization in the well-known 
old remnant after the recent nova eruption in 2011 demonstrated that its 3-dimensional shape is dominated by an inclined torus [98, 87]. And as with V959 Mon, long-slit spectroscopy of the old shell by [64] suggests that the inclined torus is part of a more complete, spherical shell. Once the ejecta from the 2011 eruption had had a few years to expand, HST/WFC3 images showed that they also contained a central core consistent with an inclined torus (Figure 4; the central torus is most clearly visible in the latest images, at the bottom of the figure); kinematics of the central structure from STIS spectroscopy indicate that the torus is inclined in the same direction as the torus in the old remnant and expanding with a similar velocity ([100], based on a comparison with the proper motion of knots in the old remnant measured by [80]). Moreover, our HST/STIS images show that, as in V959 Mon, the equatorial torus appears to have mechanically shaped a faster flow into bipolar lobes (although they are much fainter in T Pyx than in V959 Mon). The lobes are not easily recognizable in $H S T$ images of the old remnant [86, 80]. However, [85] reported an "extended envelope" component (in $\mathrm{H} \alpha+[\mathrm{NII}]$ ) in the remnant that could perhaps have been from faster, bipolar lobes.

Fig. 4 also shows that the ejecta from T Pyx comprised additional features besides the equatorial ring and bipolar lobes. HST resolved two bright knots that we will refer to as jet knots from 2013 June onward (in [OIII], although bipolar structure was already evident in the spatially resolved STIS spectra from 2012 July), within the eastern and western lobes. Radial velocities of approximately $\pm 800 \mathrm{~km} \mathrm{~s}^{-1}$ from STIS spectroscopy combined with proper motions (and taking $\mathrm{d}=4.8 \mathrm{kpc}$; [98]) indicate that they moved away from the central binary with a velocity of approximately $2000 \mathrm{~km} \mathrm{~s}^{-1}$. They thus moved with a speed of more than twice the average speed of material in the equatorial torus. Furthermore, if the jet knots moved perpendicular to the orbital plane, then the inclination of the binary must be on the high side of reported range of values. Moreover, the resemblance between the bright jet knots in T Pyx and the relatively fainter knots within the lobes of V959 Mon supports conclusions from previous studies that nova remnants have other pervasive features in addition to equatorial rings and bipolar lobes. We will describe the HST WFC3 and STIS observations of T Pyx in detail in a forthcoming paper [100].

Secondary features. Various imaging studies of nova remnants have led to the determination that many remnants contain not only bipolar lobes and equatorial rings, but also 'polar blobs', 'polar caps', 'polar rings', and knots with tails. Here we speculate that these secondary features might all be related to the main features in our HST images of the young (few-year-old) remnants of V959 Mon and T Pyx - if we take into account the possibility that most of the ejecta mass could be expelled somewhat later than the initial 'puff' of material at $t_{0}$ (the time of the TNR). Strong evidence for a delay of on the order of a month between the TNR and the expulsion of most of the mass in both V959 Mon and T Pyx comes from the evolution of the radio and X-ray emission from shock-heated plasma ${ }^{3}[61,8,37]$. In T Pyx, the delayed ejecta clearly overtook and collided with slightly slower-moving material ([89] detected P Cyg absorption features during the first few weeks with radial velocities of -1000 to $-1500 \mathrm{~km} \mathrm{~s}^{-1}$ ) about two months after the start of the eruption [61, 8]. As discussed by [6], the collision between an initial spherical shell and a

\footnotetext{
${ }^{3} \mathrm{~A}$ delay between the TNR and the expulsion of much of the ejecta raises questions about the origin of this delay. If the shock power of a few times $10^{38} \mathrm{erg} \mathrm{s}^{-1}[45,46]$ is a reasonable estimate of the rate at which the fast flow transfers energy to the slower component of the ejecta, it is important that it would take the fast flow $10^{44} \mathrm{erg} / 10^{38} \mathrm{erg} \mathrm{s}^{-1} \approx$ weeks to unbind $\sim 10^{-4} M_{\odot}$ of material sitting $\sim 10^{11} \mathrm{~cm}$ (the size scale of the binary) away from the WD?
} 


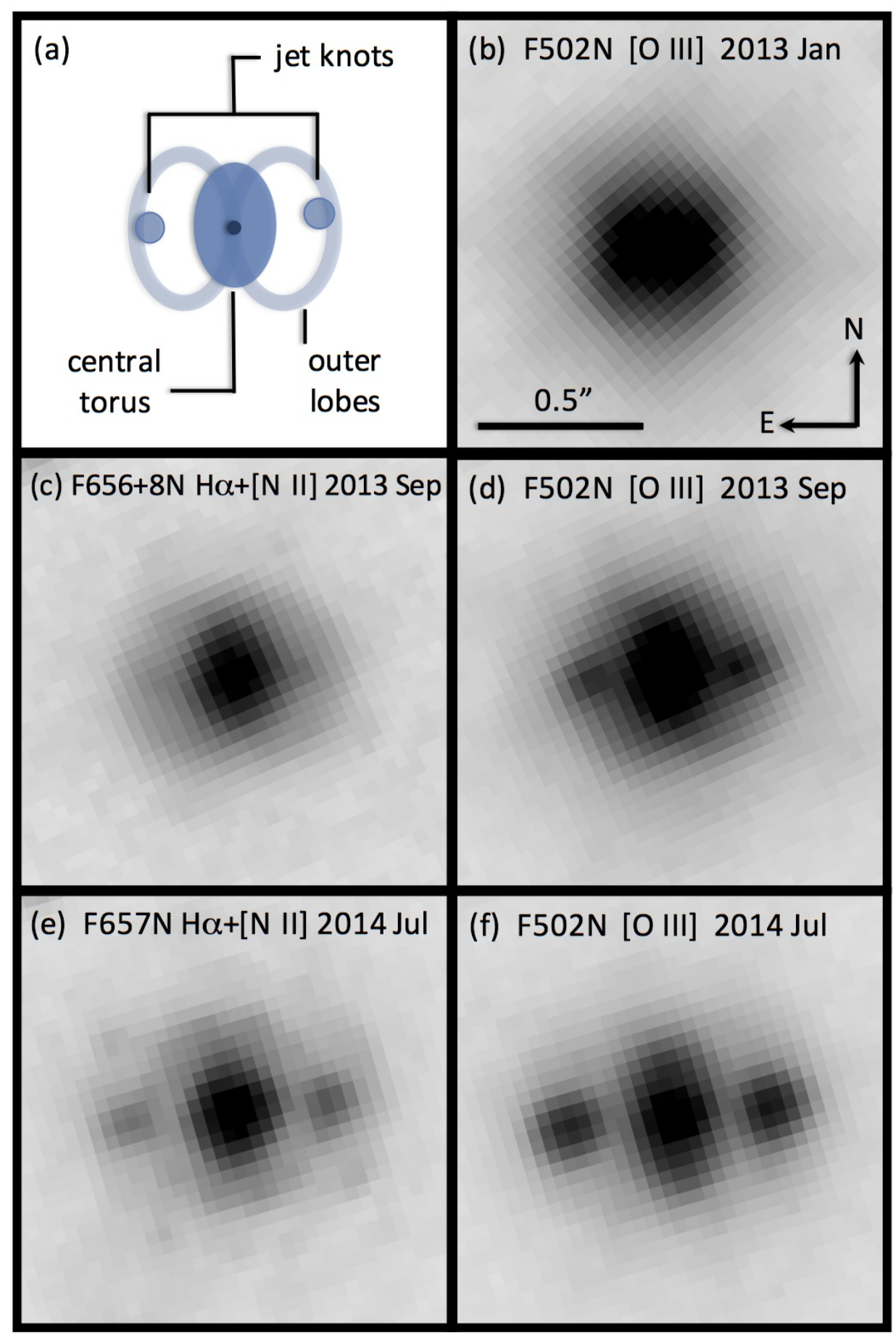

Figure 4: HST/WFC3 imaging of T Pyx. All frames are 34 pixels $=1.3^{\prime \prime}$ square, $\mathrm{N}$ up, with logarithmic intensity scaling and identical stretch (pegged to brightest pixel in F502N image in each epoch/row). (a) A to-scale schematic of the potential ejecta structures. (b) F502N 2013 January. (c) A sum of F656N+F658N 2013 September (d) F502N 2013 September. This epoch best shows the outer lobes and first reveals the jet knots. (e) F657N 2014 July, this filter provides complete velocity sampling of all components in $\mathrm{H} \alpha+[\mathrm{N}$ II]. (f) F502N 2014 July. This latest epoch shows sigpificant proper motion in the jet knots. 
later, faster bipolar flow naturally leads to polar caps, blobs, or rings. And some novae experience even more than two episodes of mass ejection; V1369 Cen experienced a series of ejections, each expelling material with velocities than the previous one (F. Walter, private communication). In any case, the jet knots in T Pyx, and the analogous features in V959 Mon, could easily be described as polar blobs (especially once the bipolar lobes faded). Finally, with faster flows overtaking slower flows at multiple locations within a given remnant, there are plenty of opportunities for instabilities to produce knots.

Implications of flow structure on $\gamma$-rays. Observations suggesting that the ejecta from novae fundamentally consist of two colliding flows - a slow flow with an equatorial density enhancement and a faster flow that is shaped by the slow flow — have bearing on $\gamma$-ray production and mass ejection in these events. For starters, if the V959 Mon scenario of $\gamma$-ray production is widespread, then $\gamma$-rays are expected to be strongest from systems with slow (less than approximately $1000 \mathrm{~km} \mathrm{~s}^{-1}$ ), radiative shocks. Such shocks in novae have high enough densities and low enough expansion speeds to efficiently trap relativistic protons, which then collide with non-relativistic protons to produce pions that decay and emit $\gamma$-rays $[45,46]$. Work by [46] showed this so-called hadronic scenario for the production of $\gamma$-rays in novae to be favored over the leptonic scenario of inverse Compton scattering by relativistic electrons. They argued that because the neutral material ahead of the forward shocks can efficiently reprocess X-rays from shock-heated plasma into the optical (at the early times when $\gamma$-rays are being produced), the ratio of $\gamma$-ray to optical fluxes places constraints on the efficiency of particle acceleration. Such a treatment constrains the particle acceleration efficiencies for both V1324 Sco and V339 Del to have been quite high and therefore more consistent with hadronic than leptonic scenarios. Other surprising results from the models explored by $[44,45,46,107,108]$ are that the observed $\gamma$-ray luminosities require shocks in novae to be very powerful (with power comparable to the Eddington luminosity for a white dwarf), and that shocks probably convert the kinetic energy of the fast flow into optical emission for at least a few weeks around maximum optical light.

Common-envelope interaction. By highlighting the importance of material concentrated in the orbital plane, recent investigations of $\gamma$-rays from novae have resurrected past questions about the role of the donor star in unbinding the WD envelope after the TNR (see, e.g., [8]). In other words, is common-envelope evolution important in shaping and/or ejecting the remnants of classical novae (e.g., [39])? Whether or not common-envelope effects are at play in classical novae, this mechanism is unlikely to be important for wide, symbiotic binaries (which give rise to embedded novae) because their donor stars are far from their eruptive WDs. So, does the lack of interaction between the expanded WD photosphere and its binary companion lead to lower ejection efficiency and therefore lower ejecta masses in some wide, symbiotic binaries? If so, could this difference help explain how quasi-steady shell burning can persist for up to centuries after novae in some symbiotic stars (such as AG Peg [76, 103]). Regardless of the answers to these questions, the flow structure, $\gamma$-ray production, and ejection of the WD envelope seem to be strongly linked.

Pre-maximum halts, THEAs, and dust. Finally, new understanding of the flow structure and shocks in novae may also have implications for the shape of optical light curves (including pre-maximum halts), the transient heavy element absorbing gas (THEA features in optical spectra; [112]), and the formation of dust. Although [25] propose the pre-maximum halts are due to changes in convective energy transfer, and this effect might well have an impact on optical light curves, the 
multi-component flow structure also naturally leads to a 'pre-maximum halt' (as is sometimes observed in optical light curves; [26]). If the initial optical rise is due to the expansion of an optically thick photosphere near the outermost edge of the ejecta, then the optical light curve must turn over or halt as this material becomes optically thin. During the halt, the optical (pseudo) photosphere recedes with respect to the outer edge of the expanding ejecta. When it reaches the slow, dense core, with its greater optical depth, the optical brightness would be expected to rise a second time along with the increase in size of the expanding core (especially if the optical brightness of the core is high due to shock heating). The optical brightness peaks when the core reaches its maximum size before beginning to become optically thin. Near optical maximum, one might expect to see blueshifted absorption by the material in the (slow) core along with emission from the optically thin fast flow. Such absorption features, with blueshifted velocities of several hundred $\mathrm{km} \mathrm{s}^{-1}$, have been observed and described as THEA lines (by [112]). With regard to the long-standing question of how dust can form in the harsh environment of a nova, [11] have recently shown that the dense, cool gas behind the type of radiative shock needed for $\gamma$-ray production also provides an ideal environment for the creation of dust grains. Thus, new understanding of the structure and microphysics of colliding flows within the ejecta from novae may help resolve several long-standing problems as well as explain the production of $\gamma$-rays.

\section{Conclusions}

\subsection{Symbiotic stars}

- We define a WD symbiotic star as a binary in which a red giant transfers enough material to a WD for the interaction to produce an observable signal at some waveband.

- The presence or absence of quasi-steady shell burning sets the luminosity of the accreting WD in a symbiotic and affects its appearance across the electromagnetic spectrum. We refer to symbiotic stars with and without shell burning on the surface of their WDs as burning and non-burning symbiotic stars, respectively.

- Non-burning symbiotic stars are difficult to find in optical spectroscopic surveys. Because their WD masses, mass transfer rates, space densities, and other properties are likely to differ from those of burning symbiotics, alternative types of searches are needed to draw accurate conclusions about the nature and evolution of this class of wide binaries.

- Non-burning symbiotics can be identified in the X-rays, by their UV excess, and by their UV variability.

- Non-burning symbiotics offer a clearer view of the accretion flow from the red giant to the WD than burning symbiotics.

\subsection{Novae}

- The pervasive production of $\gamma$-ray emission shows that shocks are common and important during nova eruptions whether they are embedded or not. The emission of $\gamma$-rays by nonembedded (classical) novae requires complex outflows that result in internal shocks. 
- The basic structure of ejecta consists of a core plus halo. Early images of V959 Mon and T Pyx share common characteristics of equatorial rings and bipolar lobes that extend to larger angular sizes, even though these novae are dissimilar in many ways. It is likely that the core has an equatorial enhancement, which interacts with the faster flow (halo) and shapes it bipolar lobes, not only in these novae but most others.

- By three and a half years after the start of the eruption, the fast component of the V959 Mon had faded almost beyond detectability. Images of older nova shells might show primarily the slow component, which is more massive and denser than the faster component.

- Collisions between the slow-moving material that comprises the core and the faster flow produce strong shocks that are crucial for accelerating particles and generating gamma-rays. The same shocks produce non-thermal radio emission, thermal X-rays, and perhaps a large fraction of optical light, at different phases of the eruption.

- Now that they have been recognized as $\gamma$-ray sources, novae serve as unique laboratories for the study of particle acceleration. Multiwavelength observations contemporaneous with gamma-ray detection have the potential to allow us to distinguish between leptonic and hadronic processes, and to constrain the shock properties, including the efficiency of particle acceleration.

\section{Acknowledgments}

We are grateful for conversations with J. Mikołajewska, T. Nelson, G. J. M. Luna, A. Lucy, M. Rupen, L. Chomiuk, and the other members of the ENova collaboration. G. J. M. Luna and A. Lucy gave helpful comments on this manuscript. The authors acknowledge support from HST grants GO-13400, GO-13796, and GO-13715, as well as NNX15AF19G and AST-1616646.

\section{References}

[1] The Fermi-LAT Collaboration, A. A. Abdo et. al. 2010, Science, 329, 817.

[2] The Fermi-LAT Collaboration, M. Ackermann, et al. 2014, Science, 345, 554.

[3] C. C. Cheung, et al. 2012, ATel\# 4224.

[4] C. C. Cheung, P. Jean, and S. N. Shore 2014, ATel\# 5879.

[5] C. C. Cheung, et al. 2016, ApJ, 826, 142.

[6] S. M. Chită, et al. 2008, $A \& A, 488$, L37.

[7] D. Chochol et al. 1997 A\&A, 318, 908.

[8] L. Chomiuk, et al. 2014, Nature, 514, 339.

[9] R. L. M. Corradi et al. 2008, $A \& A, 480,409$.

[10] R. L. M. Corradi et al. 2010, A\&A, 509, A41.

[11] A. M. Derdzinski, B. D. Metzger, and D. Lazzati 2016, arXiv:1610.02401. 
[12] M. de Val-Borro, M. Karovska, and D. Sasselov 2009, ApJ, 700, 1148.

[13] D. Dobrzycka, S. J. Kenyon, and A. A. Milone 1996, AJ, 111, 414.

[14] L. Ducci, et al. 2016, $A \& A, 592$, A58

[15] S. P. S. Eyres et al. 2005, MNRAS, 358, 1019.

[16] R. N. C. Eze, G. J. M. Luna, and R. K. Smith 2010, ApJ, 709, 816.

[17] J. Frank, A. King, and D. J. Raine 2002, Accretion Power in Astrophysics: Third Edition, (Cambridge University Press).

[18] D. K. Galloway and J. L. Sokoloski 2004, ApJ, 613, L61.

[19] R. D. Gehrz et al. 2015, ApJ, 812, 132.

[20] C. D. Gill and T. J. O’Brien, MNRAS, 300, 221.

[21] C. D. Gill \& T. J. O’Brien 1999, MNRAS, 307, 677.

[22] C. D. Gill and T. J. O'Brien, MNRAS, 314, 175.

[23] M. Gromadzki, M. Mikołajewski, T. Tomov, L. Bellas-Velidis, A. Dapergolas, and C. Gałan 2006, AcA, 56, 97.

[24] I. Hachisu and M. Kato 2001, ApJ, 558, 323.

[25] Y. Hillman, D. Prialnik, A. Kovetz, M. M. Shara, and J. D. Neill 2014, MNRAS, 437, 1962.

[26] R. Hounsell et al. 2010, ApJ, 724, 480.

[27] J. B. Hutchings 1972, MNRAS, 158, 177.

[28] K. Iłkiewicz, J. Mikołajewska, K. Stoyanov, A. Manousakis, and B. Misalski 2016, MNRAS, 462, 2695.

[29] M. Karovska et al. 2010, ApJ, 710, L132.

[30] E. Kellogg, J. A. Pedelty, and R. G. Lyon 2001, ApJ, 563, L151.

[31] E. Kellogg et al. 2007, ApJ, 664, 1079.

[32] J. A. Kennea, et al. 2009, ApJ, 701, 1992.

[33] S. J. Kenyon, The Symbiotic Stars, Cambridge University Press, New York 1986.

[34] C. Knigge, A. R. King, and J. Patterson 2000, A\&A, 364, L75.

[35] E. A. Kolotilov 1980, SvAL, 6, 268.

[36] M. I. Krauss et al. 2011, ApJ, 739, L6.

[37] J. D. Linford et al. 2015, ApJ, 805, 136.

[38] M. Livio and B. Warner 1984, Obs, 104, 152.

[39] M. Livio, A. Shankar, A. Burkert, and J. W. Truran 1990, ApJ, 356, 250.

[40] A. Lucy, et al. 2017, in preparation.

[41] G. J. M. Luna and J. L. Sokoloski 2007, ApJ, 671, 741.

[42] G. J. M. Luna, J. L. Sokoloski, and K. Mukai 2008, in RS Ophiuchi (2006) and the Recurrent Nova Phenomenon, ASP Conf. Ser., 401, 342 
[43] G. J. M. Luna, J. L. Sokoloski, K. Mukai, and T. Nelson, $A \& A$, 559, A6.

[44] P. Martin and G. Dubus 2013, A\&A, 551, A37.

[45] B. D. Metzger et al. 2014, MNRAS, 442, 713.

[46] B. D. Metzger, T. Finzell, I. Vurm, R. Hascoët, A. M. Beloborodov, and L. Chomiuk, 2015, MNRAS, $450,2739$.

[47] J. Mikołajewska, M. Friedung, S. J. Kenyon, R. Viotti 1988, The Symbiotic Phenomenon, Astrophysics and Space Science Library Vol. 145.

[48] J. Mikołajewska 2003, in Symbiotic Stars Probing Stellar Evolution, ASP Conf. Series, 303, 9.

[49] J. Mikołajewska, N. Caldwell, and M. M. Shara 2014, MNRAS, 444, 586.

[50] S. Mohamed, Ph. Podsiadlowskin 2007, in 15th European Workshop on White Dwarfs, ASP Conf. Ser., 372, 397.

[51] K. Mukai and M. Ishida 2001, ApJ, 551, 1024.

[52] K. Mukai, A. Kinkhabwala, J. R. Peterson, S. M. Kahn, and F. Paerels 2003, ApJ, 586, L77.

[53] K. Mukai, et al. 2016, MNRAS, 461, L1

[54] K. Mukai 2017, submitted to PASP.

[55] U. Munari and T. Zwitter 2002, A\&A, 383, 188.

[56] U. Munari et al. 2016, arXiv:1607.06309.

[57] K. Morihana, et al. 2016, PASJ, 68 (4), 57.

[58] U. Mürset, H. Nussbaumer, H. M. Schmid, and M. Vogel, A\&A, 248, 458.

[59] U. Mürset, B. Wolff, and S. Jordan 1997, A\&A, 319, 201

[60] T. J. Nelson, D. Donato, K. Mukai, J. L. Sokoloski, and L. Chomiuk 2012, ApJ, 748, 43.

[61] T. J. Nelson, et al. 2014, ApJ, 785, 78.

[62] J. S. Nichols, et al. 2007, ApJ, 660, 651.

[63] N. E. Nuñez, T. Nelson, K. Mukai, J. L. Sokoloski, and G. J. M. Luna 2016, ApJ, 824, 23.

[64] T. J. O’Brien and J. G. Cohen 1998, ApJ, 498, L59.

[65] S. Orlando and J. J. Drake 2012, MNRAS, 419, 2329.

[66] S. Orlando, J. J. Drake, and M. Miceli 2017, MNRAS, 464, 5003.

[67] M. Orio, et al. 2007, ApJ, 661, 1105.

[68] M. Orio, V. Rana, K. L. Page, J. L. Sokoloski, and F. Harrison 2015, MNRAS, 448, L35.

[69] B. Paczyński and A. N. Żytkow 1978, ApJ, 222, 604.

[70] K. L. Page et al. 2013, ApJ, 768, L26.

[71] D. Pandel et al. 2005, ApJ, 626, 396.

[72] Paresce et al. 1995, ApJ, 442, L57.

[73] J. Patterson, et al. 2017, MNRAS, 466, 581.

[74] T. P. Prabhu \& G. C. Anupama 1987, Ap\&SS, 131, 479. 
[75] T. P. Prabhu \& G. C. Anupama 1987, JApA, 8, 369.

[76] G. Ramsay, J. L. Sokoloski, G. J. M. Luna, and N. E. Nuñez 2016, MNRAS, 461, 3599.

[77] V. A. R. M. Ribeiro et al. 2011, MNRAS, 412, 1701.

[78] V. A. R. M. Ribeiro, U. Munari, and P. Valisa 2013, ApJ, 768, 49.

[79] R. Sahai, J. Sanz-Forcada, C. Sánchez Contreras, and M. Stute 2015, ApJ, 810, 77.

[80] B. E. Schaefer, A. Pagnotta, and M. M. Shara 2010, ApJ, 708, 381.

[81] G. H. Schaefer et al. 2014, Nature, 515, 234.

[82] E. R. Seaquist, A. R. Taylor, and S. Button 1984, ApJ, 284, 202.

[83] E. R. Seaquist and A. R. Taylor 1990, ApJ, 349, 313.

[84] E. R.. Seaquist, M. Krogulec, and A. R. Taylor 1993, ApJ, 410, 260

[85] M. M. Shara, A. F. Moffat, R. E. Williams, and J. G. Cohen, ApJ, 337, 720.

[86] M. M. Shara, et al. 1997, AJ, 114, 258

[87] M. M. Shara, et al. 2015, ApJ, 805, 148.

[88] K. J. Shen and L. Bildsten 2007, SpJ, 660, 1444.

[89] S. N. Shore, T. Augusteijn, A. Ederoclite, and H. Uthas, $A \& A$, 533, L8.

[90] S. N. Shore et al. 2013, $A \& A, 559$, L7.

[91] S. N. Shore et al. 2013, $A \& A$, 553, A123.

[92] A. Skopal 2005, A\&A, 440, 995.

[93] J. L. Sokoloski, L. Bildsten, and W. C. G. Ho 2001, MNRAS, 326, 553.

[94] J. L. Sokoloski and S. J. Kenyon 2003, ApJ, 584, 1021.

[95] J. L. Sokoloski and S. J. Kenyon 2003, ApJ, 584, 1027.

[96] J. L. Sokoloski 2003, in Symbiotic Stars Probing Stellar Evolution, ASP Conf. Proc., 303, 202.

[97] J. L. Sokoloski and L. Bildsten 2010, ApJ, 723, 1188.

[98] J. L. Sokoloski, A. P. S. Crotts, S. Lawrence, and H. Uthas2013, ApJ, 770, L33.

[99] J. L. Sokoloski et al., 2018, in preparation.

[100] J. L. Sokoloski et al., 2018, in preparation.

[101] J. Solf 1983, ApJ, 273, 647.

[102] M. Stute, G. J. M. Luna, and J. L. Sokoloski 2011, ApJ, 731, 12.

[103] T. V. Tomov, K. A. Stoyanov, and R. K. Zamanov 2016, MNRAS, 462, 4435.

[104] H. Uthas, C. Knigge, and D. Steeghs 2010, MNRAS, 409, 237.

[105] M. van den Berg, et al. 2006, ApJ, 647, L135.

[106] K. Viironen, et al. 2009, $A \& A, 502,113$.

[107] , A. Vlasov, I. Vurm, and B. D. Metzger 2015, MNRAS, 463, 394.

[108] I. Vurm and B. D. Metzger 2016, arXiv:1611.04532. 
[109] J. H. S. Weston, et al. 2016, MNRAS, 457, 887.

[110] J. H. S. Weston, et al. 2016, MNRAS, 460, 2687.

[111] J. H. S. Weston, 2016, Radio observations as a tool to investigate shocks and asymmetries in accreting white dwarf binaries, $\mathrm{PhD}$, Columbia University, Department of Astronomy.

[112] R. Williams, E. Mason, M. Della Valle, and A. Ederoclite 2008, ApJ, 685, 451.

[113] W. M. Wolf, L. Bildsten, J. Brooks, and B. Paxton 2013, ApJ, 777, 136.

[114] P. A. Woudt, et al. 2009, ApJ, 706, 738.

[115] R. Zamanov et al. 2015, MNRAS, 450, 3958.

[116] R. Zamanov, M. F. Bode, V. Stanishev, and J. Marti 2004, MNRAS, 350, 1477. 